\title{
Fracture prediction with a material model based on stress-rate dependency related with non-associated flow rule
}

\author{
Tetsuo Oya ${ }^{1, a}$, Jun Yanagimoto ${ }^{2}$, Koichi Ito $^{3}$, Gen Uemura $^{3}$ and Naomichi Mori ${ }^{3}$ \\ ${ }^{1}$ Keio University, Graduate School of Science and Technology, 2238522 Hiyoshi 3-14-1, Kohoku-ku, Yokohama, Japan \\ ${ }^{2}$ The University of Tokyo, Institute of Industrial Science, 1538505 Komaba 4-6-1, Meguro-ku, Tokyo, Japan \\ ${ }^{3}$ M \& M Research, 1500001 Jingumae 4-15-3, Shibuya-ku, Tokyo, Japan
}

\begin{abstract}
In this paper, a novel material model for anisotropic metals is proposed. Fracture prediction using the proposed model is based on stress-rate dependency related with non-associated flow rule in which an arbitrary order function for both the yield and plastic potential functions are used. In addition, an explicit formulation of the equivalent plastic strain increment that is plastic-work-conjugated with the equivalent stress is also presented. Then, fracture prediction analyses are conducted by using the $3 \mathrm{D}$ local bifurcation theory.
\end{abstract}

\section{Introduction}

Modern demand for material models is various and high level; therefore, more precise and physically valid material model has been required. Fracture prediction for sheet and bulk metal forming is one of such demands from industry and this paper focuses on it. To conduct reliable fracture prediction during metal forming, a material model that has high physical resolution should be constructed and used together with objective fracture criterion. For this purpose, a novel material model for anisotropic metals has been developed based on the nonassociated flow rule by the authors. This paper shows its outline and application on the fracture prediction problem, which has been carried out using the 3D local bifurcation theory.

Most of the phenomenological plastic constitutive models are based on the framework called the associated flow rule, which is a type of normality rule having a yield function as its plastic potential function. In metal forming analysis, the use of the associated flow rule is not necessarily a best choice for anisotropic metals such as cold-rolled steels and aluminum alloys. In metal forming research, many material models have been studied to overcome such problems. The origin of the anisotropic material model is considered to be Hill's model [1]. This model was developed on the basis of von Mises's isotropic material model and is still frequently used because of its acceptable prediction results for most general metals.

Many phenomenological yield functions have been proposed. For example, Karafillis and Boyce [2], and Barlat and Lian [3] presented yield functions for anisotropic metals. Most of their expressions are too complex to explicitly define the equivalent plastic strain

\footnotetext{
a Corresponding author: oya@sd.keio.ac.jp
}

increment, which is work-conjugated with the equivalent stress. This is because the yield function is used to express both flow stress anisotropy and deformation anisotropy. A few researchers have proposed material models based on the non-associated flow rule. Stoughton and Yoon [4] proposed a model based on the nonassociated flow rule that adopted Hill's quadratic yield function. Recently, Safaei et al. [5] proposed a model based on the non-associated flow rule that adopted Barlat's yield function [6]. These models include disadvantages of the original yield functions they made the basis; therefore, there remains difficulties toward the practical use of non-associated flow rule models.

In this paper, a novel material model for anisotropic metals is proposed. The model is based on the nonassociated flow rule with arbitrary order function for both the yield and plastic potential functions. In addition, an explicit formulation of the equivalent plastic strain increment that is plastic-work-conjugated with the equivalent stress is also presented. Then, fracture prediction analyses are conducted by using the $3 \mathrm{D}$ local bifurcation theory.

\section{Material model}

In this section, the orthodox anisotropic plasticity model proposed by Hill, which is called Hill48 model in this paper, is briefly introduced first because its formulation is essential to our model. Then, the proposed model is introduced, which is a natural extension of Hill48 for arbitrary order functions and based on the non-associated flow rule. The definitions of the yield function, plastic potential function, and the plastic strain increment are presented. 


\subsection{Hill48 model}

To obtain a concise expression for the yield function, the following deviatoric stress vector $\boldsymbol{s}$ and flow stress anisotropy matrix $\boldsymbol{A}$ are introduced:

$$
\begin{aligned}
\boldsymbol{s} & =\left\{s_{1}, s_{2}, s_{3}, s_{4}, s_{5}, s_{6}\right\}^{T} \\
& =\left\{s_{y}-s_{z}, s_{z}-s_{x}, s_{x}-s_{y}, t_{y z}, t_{z x}, t_{x y}\right\}^{T}, \\
\boldsymbol{A} & =\left[\begin{array}{cccccc}
F & 0 & 0 & 0 & 0 & 0 \\
0 & G & 0 & 0 & 0 & 0 \\
0 & 0 & H & 0 & 0 & 0 \\
0 & 0 & 0 & 2 L & 0 & 0 \\
0 & 0 & 0 & 0 & 2 M & 0 \\
0 & 0 & 0 & 0 & 0 & 2 N
\end{array}\right],
\end{aligned}
$$

where $F, G, H, L, M$, and $N$ are Hill's anisotropic parameters. Then, the yield function $f(\sigma)$ is defined as follows:

$$
f(\boldsymbol{\sigma})=\overline{\boldsymbol{\sigma}}=\sqrt{\frac{3}{2(F+G+H)}\left(s^{T} \cdot \boldsymbol{A} \cdot s\right)},
$$

where $\bar{\sigma}$ is the equivalent stress. Using the plastic work conjugacy, the definition of the equivalent plastic strain increment can be explicitly obtained as

$$
d \varepsilon^{P}=\sqrt{\frac{2(F+G+H)}{3}\left(\boldsymbol{B} \cdot d \boldsymbol{e}^{P}\right)^{T} \cdot\left(\boldsymbol{A} \cdot \boldsymbol{B} \cdot d \boldsymbol{e}^{P}\right)},
$$

where $d \boldsymbol{e}^{P}$ is the strain increment vector. Here, the matrix $\boldsymbol{B}$ is an inverse matrix of a compliance matrix $\boldsymbol{C}$ that is obtained from the following flow rule relationship with a plastic multiplier $d \Lambda$ :

$$
d \boldsymbol{e}^{p}=\frac{\partial g(\boldsymbol{\sigma})}{\partial \boldsymbol{\sigma}} d \Lambda=C \cdot s d \Lambda
$$

\subsection{Proposed model}

After the Hill48 model, many useful material models have been developed based on the associated flow rule and provided good results in some cases; however, the intrinsic problem was not solved. Namely, in an anisotropic sheet metal, the directions of yield stress evolution and plastic strain increment do not generally coincide; in other words, the shape of the yield surface and the plastic potential, which prescribes the direction of the plastic strain increment, do not match. If a yield function is devised to exhibit good agreement with yield stresses obtained from experiments, the representation of stress anisotropy will be achieved but that of deformation anisotropy will be lost. Therefore, the authors consider it is natural to adopt the non-associated flow rule to represent two different anisotropy independently [7].

Following the formulation of the above-explained Hill's anisotropic model, the proposed model is developed.
A vector $\boldsymbol{s}_{m}$, which is a deviatoric stress vector with its components having powers of $m$, is defined.

$$
\begin{aligned}
\boldsymbol{s}_{m} & =\left\{s_{1}^{m}, s_{2}^{m}, s_{3}^{m}, s_{4}^{m}, s_{5}^{m}, s_{6}^{m}\right\}^{T} \\
& =\left\{\left(\sigma_{y}-\sigma_{z}\right)^{m},\left(\sigma_{z}-\sigma_{x}\right)^{m},\left(\sigma_{x}-\sigma_{y}\right)^{m}, \boldsymbol{\tau}_{y z}^{m} \boldsymbol{\tau}_{z x}^{m}, \boldsymbol{\tau}_{x y}^{m}\right\}^{T} .
\end{aligned}
$$

Using the same anisotropic matrix $\boldsymbol{A}$ as that in the Hill48 model, the quadratic form $\boldsymbol{s}_{m} \cdot \boldsymbol{A} \cdot \boldsymbol{s}_{m}$ is obtained. This takes the form of Hill's second-order yield function with its components having powers of $2 \mathrm{~m}$. In the proposed model, we define the yield function $f(\sigma)$ as being equal to the equivalent stress $\bar{\sigma}$; namely, the expression is

$$
f(\boldsymbol{\sigma})=\bar{\sigma}=\sqrt[2 m]{\frac{3}{2(F+G+H)}\left(\boldsymbol{s}_{m}^{T} \cdot \boldsymbol{A} \cdot \boldsymbol{s}_{m}\right)} .
$$

This higher-order function preserves the form of Hill's quadratic yield function, that is, it contains the same anisotropic parameters $F, G, H, L, M$, and $N$. This feature is important because it is possible to construct a higherorder yield function by changing the power $m$ without increasing the number of undetermined variables. Therefore, the burden of determining anisotropic parameters through numerous experiments can be avoided.

In our non-associated flow rule, a function different from the yield function is adopted as a plastic potential function, which provides the direction of the plastic strain increment of the subsequent state of current stress. In this paper, the previously introduced function $f(\sigma)$ is used as the yield function, and another function $g(\boldsymbol{\sigma})$ that takes the same form as $f(\boldsymbol{\sigma})$ but has different anisotropic parameters $F^{*}, G^{*}, H^{*}, L^{*}, M^{*}$, and $N^{*}$ is adopted in the plastic potential function. In the expression, asterisks are used to distinguish $f(\boldsymbol{\sigma})$ from $g(\boldsymbol{\sigma})$. For example, the anisotropy matrix $\boldsymbol{A}$ is changed to $\boldsymbol{A}^{*}$, in which the original parameters $F, G, H, L, M$, and $N$ are also changed to $F^{*}, G^{*}, H^{*}, L^{*}, M^{*}$, and $N^{*}$, respectively. To express another order of the function, the power variable $n$ is used instead of $m$. Note that the parameters $m$ and $n$ are positive integer in this study. Thus, the plastic potential function of the proposed model takes the form of

$$
g(\boldsymbol{\sigma})=\bar{\sigma}^{*}=\sqrt[2 n]{\frac{3}{2\left(F^{*}+G^{*}+H^{*}\right)}\left(s_{n}^{T} \cdot A^{*} \cdot s_{n}\right)} .
$$

In the next subsection, how to determine the both set of anisotropic parameters is described.

From the definition of the plastic work, an explicit expression for our equivalent plastic strain increment is obtained as

$$
d \varepsilon^{P}=\frac{n \bar{\sigma}^{*_{n}}}{\bar{\sigma}} \sqrt{\frac{2\left(F^{*}+G^{*}+H^{*}\right)}{3}\left(B_{n}^{*} \cdot d \boldsymbol{e}^{P}\right)^{T} \cdot\left(\boldsymbol{A}^{*} \cdot \boldsymbol{B}_{n}^{*} \cdot d \boldsymbol{e}^{P}\right)} .
$$

\subsection{How to determine anisotropic parameters}


The main disadvantage of the non-associated flow rule models would be the increase in the number of unknown variables. Usually, these variables can be specified from experiments such as tensile tests; therefore, the use of the non-associated flow rule model leads to an increased burden of experiments and measurements. In addition, if a higher-order function is required, the burden increases, making this approach impractical. Thus, to enjoy the benefits of the non-associated flow rule model, an increase in the numbers of unknown variables should be avoided, and this is achieved with the proposed model.

The plastic anisotropy characteristics of materials are classified into two categories; namely, plastic flow stress anisotropy and plastic deformation anisotropy. The former and latter should be incorporated in the yield function and the plastic potential function, respectively. Consider cold-rolled metal sheets under a plane stress state. Under the plane stress condition, the number of variables is halved. The anisotropic variables that must be determined are $F, G, H$, and $N$ and $F^{*}, G^{*}, H^{*}$, and $N^{*}$. The former set expresses the yield stress anisotropy and the latter set expresses the deformation anisotropy.

The parameters about stress anisotropy, $F, G$, and $H$, are determined by the yield stresses that are obtained by tensile test in rolling direction, transverse direction, and equibiaxial test. The parameters about deformation anisotropy, $F^{*}, G^{*}, H^{*}$, and $N^{*}$, are determined by the $r$ values in the direction of rolling, diagonal, and transverse. The remaining parameter $N$ is determined by optimization using the tensile test data in the diagonal direction. Note that the order of the functions, $m$ and $n$, should be determined before these anisotropic parameters are determined because these values specify the function type, and have own physical meaning different from the anisotropic parameters.

\section{Fracture prediction theory}

In this section, three key theories adopted in this study will be briefly explained. These theories have been proposed by Ito et al. [8]. The proposed material model explained in the previous section is combined with these theories.

\subsection{D local bifurcation theory}

Based on Hill's general bifurcation theory, bifurcation occurs at when the following condition is satisfied.

$$
I[\Delta \boldsymbol{v}]=\int \Delta \boldsymbol{L}: \Delta \dot{\boldsymbol{S}} d V=0,
$$

where $\boldsymbol{v}$ is velocity field, $\boldsymbol{L}$ and $\dot{\boldsymbol{S}}$ are velocity gradient tensor and 1st Piola-Kichhoff stress tensor rate, respectively. $\dot{S}$ can be represented by the Cauchy stress tensor $\boldsymbol{\sigma}$ as

$$
\dot{S}=D: \dot{\varepsilon}+\sigma \operatorname{tr}(L)+\omega \cdot \sigma-\sigma \cdot \omega-L \cdot \sigma=A: L
$$

where $\dot{\boldsymbol{\varepsilon}}, \boldsymbol{\omega}, \boldsymbol{D}$ are strain rate tensor, spin tensor, and tangent stiffness tensor, respectively. And $\boldsymbol{A}$ is a fourth rank tensor that relates the nominal stress rate $\dot{S}$ and velocity gradient tensor $\boldsymbol{L}$. To characterize the bifurcation mode, the velocity gradient tensor is allowed to be discontinuous when the velocity gradient tensor crosses the bifurcation border $\Gamma$. $\boldsymbol{L}$ can be represented by the normal vector $\boldsymbol{n}$ on the bifurcation border and the local deformation mode vector $\boldsymbol{m}$ that is normal to $\boldsymbol{n}$

$$
L=m \otimes n .
$$

Moreover, the mode vector $\boldsymbol{m}$ can be decomposed into three different vectors. Note that the vectors $\boldsymbol{m}$ and $\boldsymbol{n}$ are different from the parameters in the yield and plastic potential functions. Substituting Eq. (11) and (12) into (10), we have

$$
I[\boldsymbol{m}, \boldsymbol{n} ; \boldsymbol{\sigma}]=h H[\boldsymbol{m}, \boldsymbol{n} ; s]-\boldsymbol{\sigma} \Sigma[\boldsymbol{m}, \boldsymbol{n} ; s]
$$

where $h$ is a hardening rate and the first and second term of this formula are described as

$$
\begin{gathered}
H[m, n ; s]=m \cdot n \cdot \bar{D}(s) \cdot n \cdot m, \\
\Sigma[m, n ; s]=\frac{1}{2}[m \cdot s \cdot m-n \cdot s \cdot n I] .
\end{gathered}
$$

In an elasto-plastic material subjected to large strain, ignoring elastic deformation, the tangent stiffness tensor can be assumed to be proportional to the hardening rate $h$. Under this assumption, the tangent stiffness tensor can be represented with the polar decomposition form.

Then, the current stress is represented by

$$
\sigma=\sigma s, \quad \sigma=\sqrt{\sigma: \sigma}, \quad s=\sigma / \sigma
$$

where $\sigma$ and $s$ are the norm of the current stress tensor and normalization tensor which gives stress ratios for each stress component, respectively.

Based on these relations, we finally have the following local bifurcation criterion.

$$
\left(\frac{\sigma}{h}\right)_{c r}=\min \left(\frac{H[\boldsymbol{m}, \boldsymbol{n} ; s]}{\Sigma[\boldsymbol{m}, \boldsymbol{n} ; \boldsymbol{s}]}\right) .
$$

\subsection{Ito-Goya's plastic constitutive model}

Local bifurcation brings abrupt change on the current strain rate direction. Since the classical $J_{2}$ theory does not allow the rotation of the strain rate direction caused by the subsequent stress rate direction, it is not appropriate to the bifurcation problems. Therefore, in this study, ItoGoya's plastic constitutive equation is applied, because this model can take the dependency of the strain rate direction on the stress rate direction into account. In this study, the revised Ito-Goya's plastic constitutive equation represented as follows was used. 


$$
d \boldsymbol{\varepsilon}^{p}=\Lambda\left(\boldsymbol{n}_{F}: \boldsymbol{l}_{p}\right)\left|d \boldsymbol{\sigma}^{\prime}\right|\left[K_{C} d \boldsymbol{l}+\left(1-K_{C}\right) \boldsymbol{n}_{N}\right],
$$

where $\boldsymbol{n}_{N}$ is an unit tensor called natural direction. This tensor indicates the direction of the deviatoric stress rate $d \boldsymbol{\sigma}^{\prime}$ that is identical to that of the plastic strain rate. The other terms introduced in this equation can be defined as:

$$
n_{F}=\frac{\nabla \overline{\boldsymbol{\sigma}}(\boldsymbol{\sigma})}{|\nabla \overline{\boldsymbol{\sigma}}(\boldsymbol{\sigma})|}, \quad n_{N}=\frac{\nabla \overline{\boldsymbol{\sigma}}^{*}(\boldsymbol{\sigma})}{\left|\nabla \bar{\sigma}^{*}(\boldsymbol{\sigma})\right|}, \quad l=\frac{d \boldsymbol{\sigma}^{\prime}}{\left|d \boldsymbol{\sigma}^{\prime}\right|}, l_{p}=\frac{\boldsymbol{\sigma}^{\prime}}{\left|\boldsymbol{\sigma}^{\prime}\right|}
$$

In Eq. (18), the parameter $K_{C}$, which takes a value between 0 and 1 , shows the dependency of the direction of the strain rate on stress rate. When $K_{C}$ is equal to 1 , the Ito-Goya's constitutive equation becomes the $J_{2}$ flow theory.

\subsection{Evaluation of sheet formability}

The bifurcation criterion represented by Eq.(17) indicates that the local bifurcation, which is specified by the mode vectors $\boldsymbol{m}$ and $\boldsymbol{n}$ that are based on the current stress ratio tensor $s$, should be identified by the ratio $\sigma / h$ that means the ratio of the stress level to the work-hardening. In another words, the formability represented in the $\sigma / h$ plane is completely free from the strain path dependency that is usually observed in a typical FLD (Forming Limit Diagram) represented in strain space. Mechanically, stress $\sigma$ means the intensity of fracture initiation, and the hardening coefficient $h$ means the material's resistance against fracture. Therefore, exhibiting FLDs on the $\sigma / h$ plane is mechanically reasonable.

The fracture limit lines in this FLD show both the SR (Stören-Rice) limit and 3D local bifurcation limit. The typical bifurcation that leads to final fracture would be lie between the lower bound represented by the SR limit and the upper bound represented by the 3D local bifurcation limit.

\section{Fracture prediction analyses}

In this section, the effect of the orders of the yield function and the plastic potential function in the proposed anisotropic plastic constitutive equation on the fracture prediction is numerically investigated. Using the constitutive relation in Eq.(18), assuming proportional loading, integrations on the stress increment is carried out from zero value with variable stress ratio $\alpha=\sigma_{2} / \sigma_{1}$. The stress ratio $\alpha$ takes values between 0 and 1, which means the stress state changes from uniaxial to equibiaxial via plane strain state. For each stress state, the functional $\boldsymbol{I}$ can be calculated, whose sign is available to judge the 3D local bifurcation criterion.

A virtual material is used for this analysis. Its material properties are as follows;

$$
\bar{\sigma}=500\left(\varepsilon^{P}\right)^{0.2}, \quad K_{C}=0.2 .
$$

The calculation results are shown in Figures 1 and 2. In these figures, the SR limits and 3D limits, calculated for each stress ratio, are plotted as upper lines and lower lines, respectively. Note that the calculated values indicate necking initiation based on each theory and we regard these values as potential onset of fracture. Since the purpose of this analysis is to investigate the effect of the order of the proposed yield and plastic potential functions on fracture prediction, an isotropic material is assumed in these calculations. When anisotropy is assumed, the shape of the yield and plastic potential function differs, which would affect the calculation and bring more complicated results.

First, the order of the plastic potential function $n$ is fixed to 2 , and that of the yield function $m$ is varied as 1 , 2, 3, and 4. The result is shown in Fig.1. It can be observed that the increase in the order of the yield function did not affect each fracture initiation predicted by both the SR theory and 3D theory; in other words, all the fracture limit curves coincided. We considered this result as follows: the order of the yield function affected both the stress $\sigma$ and hardening ratio $h$; therefore, the effect of the order was offset and the fracture limit curves became the same.

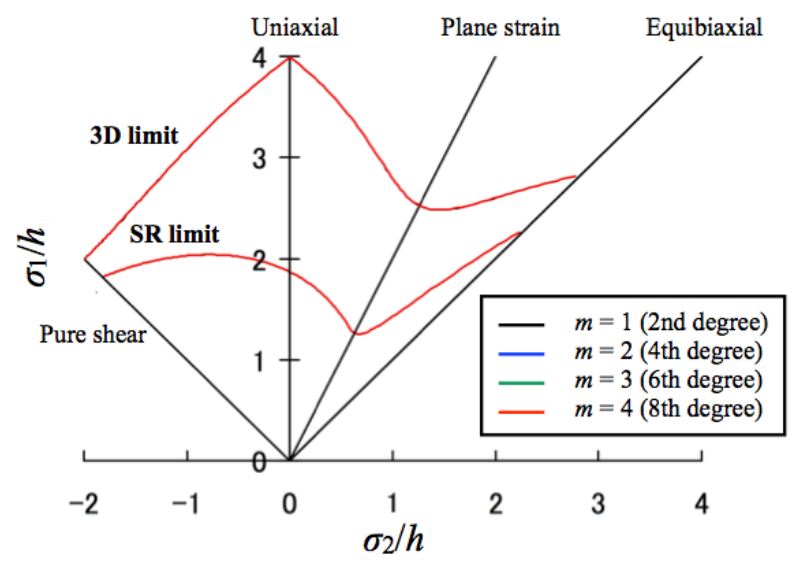

Figure 1. Predicted fracture limits for varied yield function's order; $n=2, m=1,2,3$, and 4 .

Second, the order of the yield function $m$ is fixed to 2 , and that of the plastic potential function $n$ is varied as 1,2 , 3 , and 4. The result is shown in Fig.2. In contrast to the first case, the predicted fracture limits in uniaxial, plane strain, and equi-biaxial directions are identical for any $n$; however, deteriorations are observed at in-between regions. The reason is assumed that the natural direction in Eq.(18) becomes identical in these directions for each $n$ value, which resulted in these fracture limit curves. Especially in plane strain direction, the higher-order plastic potential function brings relatively flat shape in this direction, which could be the reason for flat shape in the fracture prediction curves. 


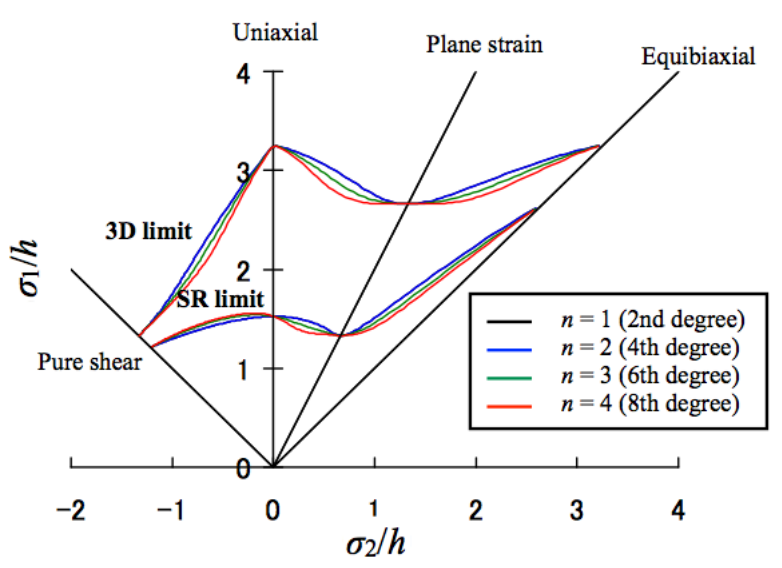

Figure 2. Predicted fracture limits for varied plastic potential function's order; $m=2, n=1,2,3$, and 4 .

Some mechanical characteristics are revealed through these simulations; however, there are many issues to be verified and considered because the effect of separation of the yield and plastic potential functions on the fracture limit prediction have never been sufficiently investigated before this research. It seems apparent that this approach will bring deep insight into the material behaviour in large deformation and near the fracture mechanism due to its considerable physical resolution; therefore, more effort should be paid to numerical and experimental investigations for this model.

\section{Conclusions}

A novel material model for anisotropic metals is proposed. The proposed model is based on the nonassociated flow rule with arbitrary order function for both the yield and plastic potential functions. In addition, an explicit formulation of the equivalent plastic strain increment that is plastic-work-conjugated with the equivalent stress is also presented. Then, fracture prediction analyses are conducted by using the 3D local bifurcation theory. Numerical simulations of the proposed model are conducted with virtual material data. Physically reasonable results were obtained by these analyses.

\section{References}

1. R. Hill, Proc. Roy. Soc. London, 197, 281-297 (1948).

2. A. P. Karafillis and M. C. Boyce, J. Mech. Phys. Solids, 41, 12, 1859-1886 (1993).

3. F. Barlat and J. Lian, Int. J. Plasticity, 5, 51-66 (1989).

4. T. B. Stoughton and J. W. Yoon, Int. J. Plasticity, 25, 1777-1817 (2009).

5. M. Safaei, S. L. Zang, M. G. Lee, and W. D. Waele, Comput. Mater. Sci., 81, 15-29 (2014).

6. F. Barlat, J. C. Brem, J. W. Yoon, K. Chung, R. E. Dick, D. J. Lege, F. Pourboghrat, S. H. Choi, and E. Chu, Int. J. Plasticity, 19, 1297-1319 (2003).
7. T. Oya, J. Yanagimoto, K. Ito, G. Uemura, and N. Mori, Procedia. Engineering, 81, 1210-1215(2014).

8. K. Ito, K. Sato, M. Goya, and T. Yoshida, Int. J. Mech. Sci., 42, 2233-2248 (2000). 